\title{
A novel formed classifier for analyzing cellular contents in cells images
}

\author{
M.A.G. López*, N.G. Posada*, J.R. Gadelha**,*** and F. Morgado** \\ * INEGI, Faculty of Engineering, University of Porto, Portugal \\ **CESAM \& Department of Biology, University of Aveiro, Portugal \\ *** CAPES Foundation, Ministry of Education of Brazil, Brasília - DF 70040-020, Brazil
}

Several approaches for cell image segmentation have been proposed and applied with success in the biomedical sciences field, but cell content estimation is still a not solved problem [1,2]. This paper presents a novel formed classifier that combine appropriately "intelligent scissors" (livewire), object's pixel information (RGB values of colors) and probabilistic artificial neural networks (ANN) techniques for segmenting and estimating precisely the cytoplasm composition in relation to protein, lipid, and carbohydrates. Based on this it is possible to estimate the gonad's state of development and the maturation stage of oocytes in adult ovigerous females gonads[3].

The ovigerous mature females (identifiable by their brood pouch - empty, with eggs, with eyeless or eyed embryos) were selected under a dissecting microscope in the laboratory. A total of 170 gonadic masses from females containing oocytes in different developmental stages were analyzed. A total of 100 sections were observed by light microscopy in order to record the arrangement of the ovaries and determine the maturity stage of the various germ cells.

With the ANN network (Fig. 1) trained is possible to carry out the classification step. Classification aims to associate each cytoplasm pixel to one of the selected classes. In this process each cytoplasm pixel is simulated (Figure 2 A). As result a new image containing the classified cytoplasm is produced, where each class is represented by a different colour level (Figure $2 \mathrm{~B}$ ). Figure $2 \mathrm{C}$ show from left to right the full "classification flow process" including ROI selection, cytoplasm and nucleus contour extraction, cytoplasm segmentation and cytoplasm content classification. The proposed classifier was tested successfully in a representative image dataset, demonstrating the effectiveness and robustness of this new method facilitating the histochemical analysis of cell contents, based on a gonads model.

\section{References}

1. Tao S. et al., IEEE Transactions on Neural Networks, 18:1424-1432, 2007.

2. Szabo B.K. et al., Academic Radiology, 11:1344-1354, 2004.

3. Kang H.W., Pattern Recognition Letters, 26:2042-2051, 2005. 


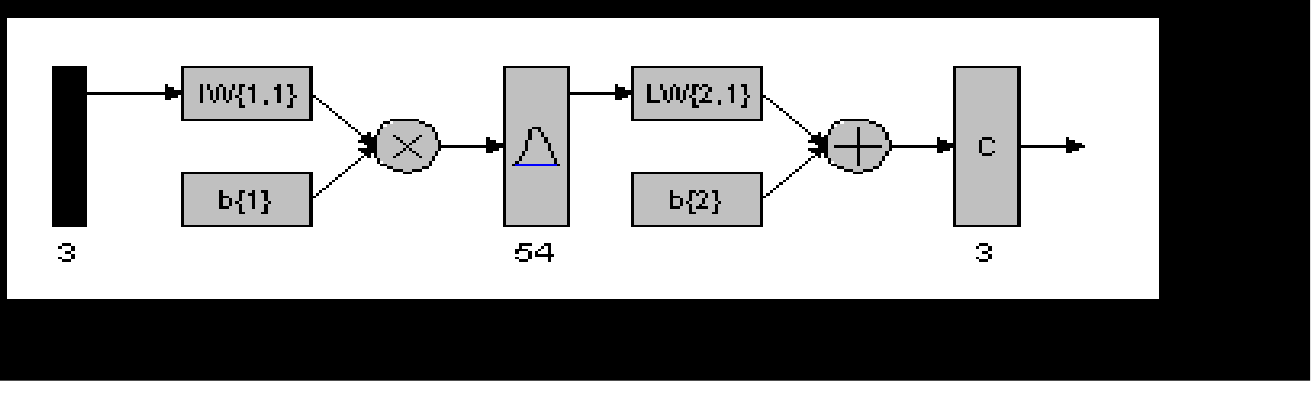

Figure 1 - Probabilistic Radial Basis ANN.

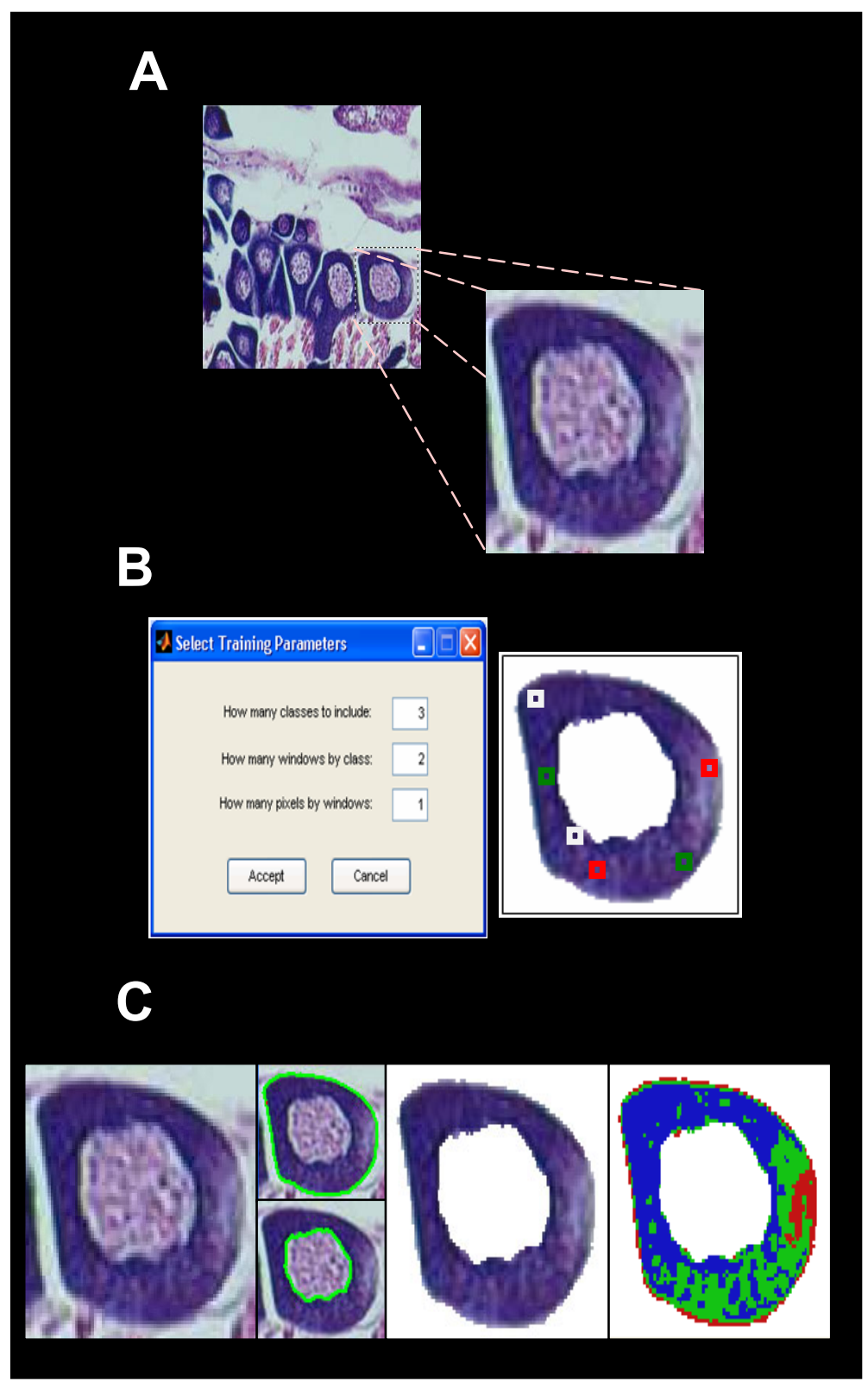

Figure 2 - (A) ROI selection; (B) Training; (C) "Classification flow process". 